\title{
Überlegene Wirksamkeit von Ixekizumab im Vergleich zu Ustekinumab
}

Bei der diesjährigen Jahrestagung der American Academy of Dermatology (AAD) zeigten neue Studiendaten zum Interleukin(IL)-17A-Inhibitor Ixekizumab zur Behandlung von Erwachsenen mit mittelschwerer bis schwerer Plaque-Psoriasis eine überlegene Wirksamkeit im Vergleich zum IL-12/23-Inhibitor Ustekinumab über einen Zeitraum von 24 Wochen.

Im Rahmen der direkten, multizentrischen, randomisierten, verblindeten Phase-III-Vergleichsstudie IXORA-S erhielten Patienten über einen Zeitraum von 52 Wochen entweder Ixekizumab oder Ustekinumab gemäß der jeweils zugelassenen Dosierung. Die auf der AAD-Tagung vorgestellten Ergebnisse über 24 Wochen zeigen für die mit Ixekizumab behandelten Patienten signifikant höhere Ansprechraten im Vergleich zu Ustekinumab. Ein PASI 90-Ansprechen erreichten unter einer Behandlung mit dem IL-17A-Inhibitor $83 \%$ der Patienten, mit Ustekinumab waren es hingegen $59 \%$.

Die Verträglichkeit von Ixekizumab Quelle: Presseaussendung Eli Lilly GmbH

Quelle: Presseaussendung MedUni Wien deutlich verbessert werden kann.
Mit Hilfe der so genannten IgEnioSäule, einem spezifischen EinwegAdsorber zur Behandlung von IgEverursachten Krankheiten, wird der IgE-Spiegel im Blut-Plasma verringert: Das Blut des Betroffenen wird durch eine "Säule" geleitet, in der die IgE-Antikörper kleben bleiben - und zwar mittels Sepharose-Kügelchen, bisherigen klinischen Studien. Die Ausprägung unerwünschter Ereignisse war in der Regel mild bis moderat, sie traten vergleichbar häufig auf wie unter einer Behandlung mit Ustekinumab.

„Menschen mit mittelschwerer bis schwerer Plaque-Psoriasis wünschen sich eine weitgehend erscheinungsfreie Haut, betonte Professor Dr. Kristian Reich, Dermatologikum Hamburg. „Mit IL-17A-Inhibitoren wie Ixekizumab können im Vergleich zu bisherigen Standardtherapien mehr Patienten eine PASI 90-Antwort oder eine erscheinungsfreie Haut erreichen. Die Daten dieser direkten Vergleichsstudie sind ähnlich denen der placebo-kon- trollierten Zulassungsstudien, in denen Patienten mit Ixekizumab ebenfalls ein hohes Maß an Erscheinungsfreiheit erreichten. In der direkten Vergleichsstudie gegen Ustekinumab war Ixekizumab an Woche 12 und Woche 24 bezüglich PASI 90 und PASI 100 signifikant überlegen. Der IL-12/23-Inhibitor ist eines der derzeit am häufigsten eingesetzten Biologika bei mittelschwerer bis schwerer Plaque-Psoriasis. Neue Therapien müssen sich heute hieran messen lassen."

hautnah $2017 \cdot 16: 36$

DOI 10.1007/s12326-017-0237-5

๑) Springer-Verlag Wien 2017

\section{Neue Methode entfernt Auslöser für allergisches Asthma}

Wissenschafter der MedUni Wien haben eine Methode entwickelt, mit der Immunglobulin E (IgE)-Antikörper aus dem Blut abgesaugt werden und so die Lebensqualität von Menschen mit schwerem allergischen Asthma

die mit lgE-Fänger Proteinen besetzt sind. Diese binden das IgE in der Säule und saugen es praktisch beim Durchfließen während der Blutwäsche ab. Einen ähnlichen Adsorber gibt es auch für lgG-Antikörper bei Autoimmunerkrankungen.

In der ersten mit IgEnio durchgeführten Studie [1] konnten die Forscher der MedUniWien am Institut für Pathophysiologie und Allergieforschung um Dr. RudolfValenta, Dr. Christian Lupinek und Dr. Kurt Derfler von der Klinischen Abteilung für Nephrologie und Dialyse, sowie Dr. Ventzislav Petkov von der Klinischen Abteilung für Pulmologie, zeigen, dass dieses Absaugen für die Betroffenen eine deutliche Verbesserung 